\title{
L'assorbimento della radiazione solare su scala planetaria $\left(^{*}\right)$
}

\author{
(The absorption of the solar radiation by the Earth)
}

\author{
R. Cialdea - S. Sciarratta $(* *)$
}

Ricevuto il 20 Giugno 1967

\begin{abstract}
Risssunto. - Nella presente nota gli $A$ A. cercano un eventuale fenomeno at andamento diumo universale che possa influenzare $i$ vari fenomeni naturali terrestri. Poichó la causa prima degli andamenti diurni è la radiazione solare che arriva sulla terra, un tale fenomeno può ricercarsi solo nel fatto che, in seguito alla rotazione diurna della terra, le regioni illuminate dal sole sono via via diverse al variare del tempo universale; infatti poichó esse hanno un albedo diverso, la radiazione solare assorbita dalla terra avrà un andamento diurno universale.

Gli AA. hanno calcolato la superficie apparente (vista dal sole) delle terre emerse e quella dei mari in varie ore del giorno ed in varie epoche dell'anno. Allo scopo poi di tener conto dell'assorbimento della radiazione solare da parte dell'atmosfera, si è ripetuto il calcolo tenendo conto di questo assorbimento. Si sono cosi trovati gli andamenti di queste superfici che rappresentano, a meno di una costante, l'energia solare che viene effettivamente assorbita dalla terra.

Dal confronto di questi andamenti con quello di uno dei fenomeni naturali ad andamento diurno universale e cioè con quello del campo elettrico terrestre, si è visto che, con delle opportune riduzioni, è possibile far coincidere questi due andamenti. Queste riduzioni consistono in un
\end{abstract}

(*) Questa nota è stata presentata al XVI Convegno dell'A.G.I. Napoli, 22-24 Maggio 1967.

(**) Istituto Nazionale di Geofisica. Cattedra di Fisica Terrestre del. l'Università - L'Aquila. 
anticipo del massimo di circa quattro ore ed in un coefficiente che tiene conto della diversita dell'albedo presentato dalle terre emerse e dai mari.

Gli $A$. riconoscono che, con questo studio preliminare, non sia pos. sibile spiegare il meccanismo con il quale l'energia assorbita complessivamente dalla terra influenzi il campo elet trico terrestre: tuttavia gli $A$ A. sottolineano l'interesse di questa correlazione, che potrì portare un contributo anche allo studio della circolazione generale dell'atmosfera.

SumMary. - In the present paper the $A$. search an eventual phenomenon with the universal diurnal variation, which may influence the natural terrestrial phenomena. Considering that the first cause of the diurnal variations is the solar radiation arriving on the Earth, such a phenomenon may be foumded in the dinmal rotation of the Earth, therefore the regions illuminated by the sun are different according to the universal time. Since the regions of the Earth have different albedo, the solar ratdiation absorbed by the entire Earth will have an universal dinmal variation.

The $\Lambda \Lambda$. have calculated the apparent surface (with the sun as a point of view) of the lands and of the seas at different hours (U.'T.) and in the different period of the year.

In order of include in computation the absorption of the solar radiation by the atmosphere, the $\Lambda A$. have calculated these surfaces with a correction due to this absorption. In these computations the eventual cloudiness not appears, because in this first approximation the cloudiness is supposed equal on the entire Earth. These surfaces with the correction of the atmospheric absorption are proportional to the solar energy really absorbed by the entire Earth.

By the comparison of the universal diurnal variation of these surfaces with that of a natural phenomenon (the atmospheric electric field), the $A$. show that it is possible, with some reductions, to make these variations coincide. These reductions are an advance of the maximum (4 hours) and a coeflicient which reckons the albedo's difference.

The $A \Lambda$. recognize that it is no possible with this preliminary study to explain the influence of the solar energy absorbed by the entire Earth on the atmospheric electric field: but according to the $\Lambda$ A. this correlation will lake an important contribution also to the researches for the general atmospheric circulation.

Il bilancio termico dell'atmosfera(') viene effettuato studiando i diversi fattori che concorrono ad esso e cioe l'albedo, l'evaporazione, lir condensazione, la conduzione ecc. Esso rappresenta un problema 
molto complesso dato il gran numero di fattori dipendenti dal tempo in modo non sempre prevedibile, per lo meno a lunga scadenza.

Lo scopo della presente nota è di vedere invece se nel bilancio termico complessivo della terra (continenti, mari, atmosfera) sia possibile trovare un qualche fattore che abbia un andamento diurno universale. Se si potesse trovare un tale fattore, esso potrebbe assumere una grande importanza perché alcuni fenomeni naturali, quale per es. il campo elettrico terrestre (), presentano un evidente andamento diurno universale.

Lá causa prima di un tale eventuale fattore può ricercarsi solo nel fatto che, in seguito alla rotazione della terra, le regioni illuminate dal sole sono via via diverse al variare del tempo universale e poiché esse Lamno un albedo diverso, l'assorbimento della radiazione solare avrà un andamento diurno universale. Orbene le terre enerse ed i mari sono quelle superfici della terra che presentano la massima diversiti di comportamento ai fini del bilancio termico. Infatti esse, oltre ad avere due albedi notevolmente diversi, si comportano in modo diverso per quanto riguarda lo scambio di energia termica con l'atmosfera. Sulle terre emerse questo scambio è dovuto alla conduzione ed alla convezione, mentre sui mari esso è dovuto principalmente ai fenomeni di evaporazione. Abbiamo pertanto pensato di calcolare la superfieie delle terre emerse e quindi quella dei mari, che istante per istante sono esposte ai raggi solari. Ma poiché ciò che interessa in definitiva è l'energia solare che cade sulle terle e quella che arde sui mari, si sono calcolate le aree delle superfici apparenti, illuminate dal sole, con una proiezione ortografica, avendo preso il sole come punto di vista. Allo scopo di avere una valutazione dell'andamento di queste aree si è diviso tutta la terma in regioni aventi le dimensioni di $10^{\circ} \times 10^{\circ}$ : si sono calcolate graficamente le aree ricoperte dalle terre amerse per ciascuna regione moltiplicandole per il coseno dell'altezzil zenitale del sole calcolata per il centro della regione stessa ad una deterninata ora (T.U.) e per vari periodi dell'ammo.

Si sono poi sommati tutti questi prodotti relativi ad ogni epoca alle regioni illuminate dal sole. Il calcolo è stato quindi ripetuto per varie ore (T.U.) e precisamente ad intervalli di 10 minuti primi per un gionno equinoziale $\left(\delta=0^{\circ}\right)$, per un giorno prossimo al solstizio estivo $\left(\delta=+20^{\circ}\right)$ e per un giorno prossimo al solstizio invernale $\left(\delta=-20^{\circ}\right)$. Questi valori $\left(+20^{\circ} \mathrm{e}-20^{\circ}\right)$ della declinazione del sole sono stati scelti per comodità di caleolo, avendo suddiviso la terra in regioni aventi un'ampiezza di $10^{\circ}$ in latitudine: ciononostante noi assume- 
remo questi andamenti come caratteristici delle epoche equinoziali e solstiziali rispettivamente. Il risultato è riportato nella Tabella I e nella Fig. 1 dove in ascisse è il T.U. ed in ordinate le aree così calcolate. Le tre curve si riferiscono agli equinozi ed ai due solstizi. Nella Fig 1 è stato riportato anche l'andamento della superficie apparente dei mari illuminati dal sole, ottenuta per differenza dalla superficie apparente di tutta la terra.

'Tab. I .. SUPERFICIE APPAREXTE

DHLLE TERRE EMERSE ILIUMINATE DAL SOLE (in $10^{4} \mathrm{~km}^{2}$ ).

\begin{tabular}{|c|c|c|c|c|c|c|c|}
\hline 'T. U. & liquinoz. & $\begin{array}{c}\text { Solstizio } \\
\text { estivo }\end{array}$ & $\begin{array}{l}\text { Solstizio } \\
\text { invernale }\end{array}$ & 'T. U. & Equinoz. & $\begin{array}{c}\text { Solstizio } \\
\text { ostivo }\end{array}$ & $\begin{array}{l}\text { Solstizio } \\
\text { invernale }\end{array}$ \\
\hline 0.40 & 1834 & 2663 & 1572 & 12.40 & 4622 & 5407 & 4071 \\
\hline 1.20 & 2127 & 2939 & 1825 & 13.20 & 4460 & 5234 & 3921 \\
\hline 2.00 & 2457 & 3243 & 2090 & 14.00 & 4264 & 5007 & 3733 \\
\hline 2.40 & 2791 & 3568 & 2340 & 14.40 & 4019 & 4713 & 3513 \\
\hline 3.20 & 3129 & 3910 & 2582 & 15.20 & 3744 & 4376 & 3277 \\
\hline 4.00 & 3490 & 4270 & 2865 & 16.00 & 3470 & 4062 & 3040 \\
\hline 4.40 & 3888 & 4659 & 3192 & 16.40 & 3237 & 3792 & 2832 \\
\hline 5.20 & 4310 & $5(072$ & 3531 & 17.20 & 3046 & 3553 & 2667 \\
\hline 6.00 & 4701 & 5480 & 3834 & 18.00 & 2862 & 3315 & 2534 \\
\hline 6.40 & 5021 & 5813 & 4078 & 18.40 & 2662 & 3069 & 2378 \\
\hline 7.20 & 5241 & 6037 & 4258 & 19.20 & 2431 & 2828 & 2180 \\
\hline 8.00 & 5325 & 6145 & 4356 & 20.00 & 2153 & 2585 & 1947 \\
\hline 8.40 & 5294 & 6150 & 4355 & 20.40 & 1852 & 2334 & 1702 \\
\hline 9.20 & 5191 & 6072 & 4312 & 21.20 & 1585 & 2138 & 1471 \\
\hline 10.00 & 5063 & 5946 & 4287 & 22.00 & 1403 & 2064 & 1296 \\
\hline 10.40 & 4954 & 5799 & 4277 & 22.40 & 1352 & 2109 & 1205 \\
\hline 11.20 & 4864 & 5664 & 4257 & 23.20 & 1433 & 2217 & 1227 \\
\hline 12.00 & 4761 & 5543 & 4191 & 24.00 & 1602 & 2437 & 1362 \\
\hline
\end{tabular}

Consideriamo ora l'andamento della superficie apparente delle terre illuminate dal sole. Esso presenta un andamento diurno abbastanza regolare con un minimo e un massimo ben pronunciati e le 
cui posizioni sono state calcolate interpolando ed approssimandono i valori ai 10 minuti primi (Tab. 2).

'lab. II

\begin{tabular}{|c|c|c|}
\hline Epoca dellamno & Massino & Minimo \\
\hline Equinozi & 8.10 & $2 \cdot 2.30$ \\
\hline Solstizio estivo & 8.20 & 22.10 \\
\hline Solstizio invernale. . . & 8.20 & 23.00 \\
\hline
\end{tabular}

Si è poi calcolato l'andamento medio annuo di questa superficie apparente. A tale scopo, in base all'osservazione che le differenze tra

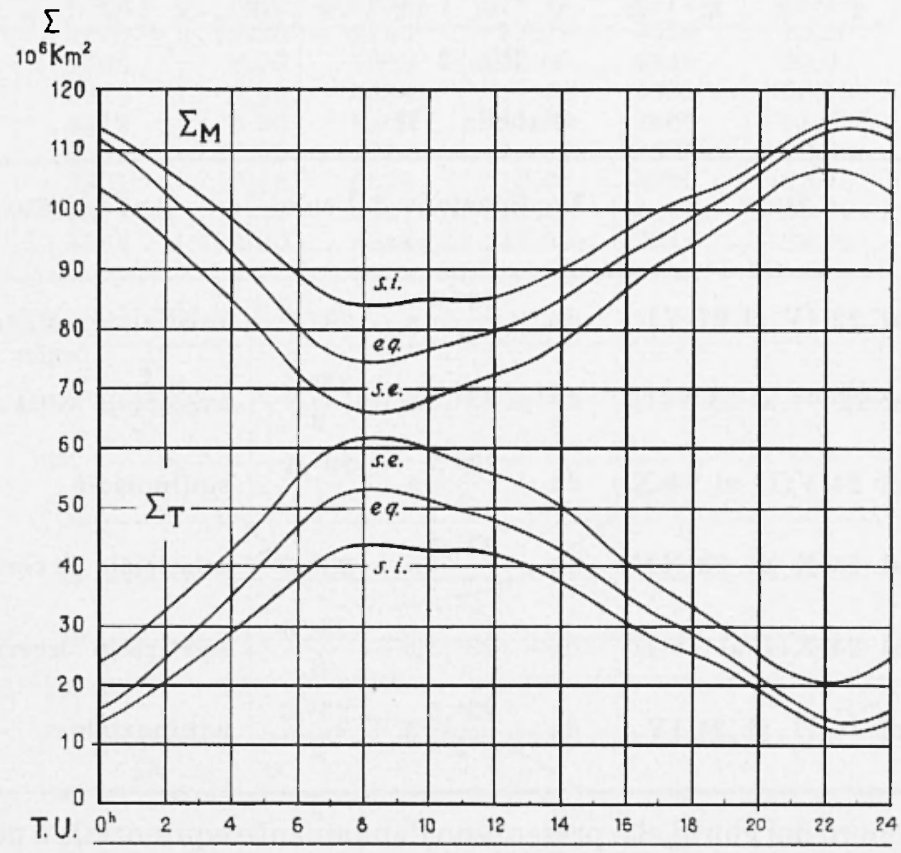

Fig. I

gli andamenti nei diversi periodi dell'anno sono dovate essenzialmente ai diversi valori della declinazione del sole, si è proceduto al callcolo dell'andamento medio nel seguente modo. La declinazione del sole 
varia durante l'anno da $+23^{\circ}, 5$ a $\ldots 23^{\circ}, 5$ (v. Fig. 2). Pertanto si è divisa quessta escursione in 6 periodi secondo la Tabella III e si è supposto che in ciascuno di questi periodi I'influenza della declinazione fosse costante, in modo da dare all'andamento delle superfici apparenti la caratteristica indicata nell'ultima colonna della Tabella stessa.

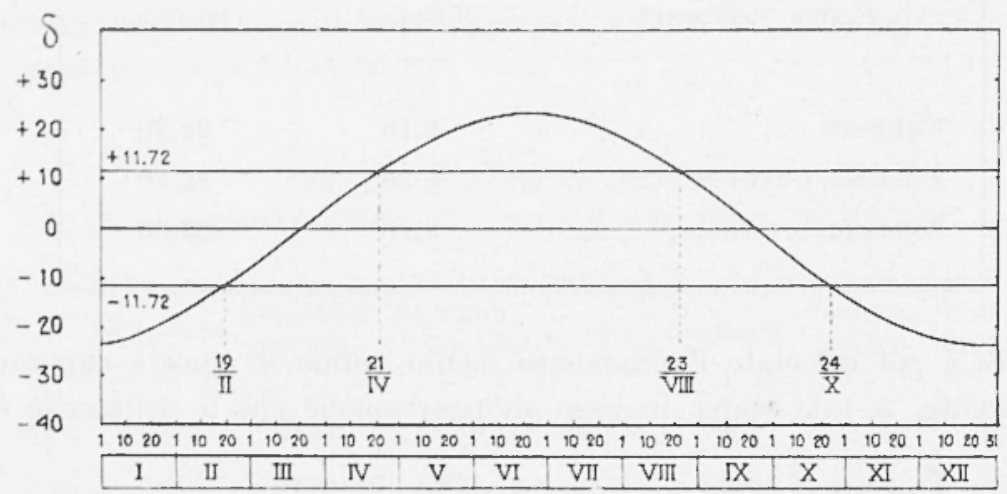

Fig. 2

Tabella III

\begin{tabular}{|c|c|c|c|}
\hline $\begin{array}{l}\text { Perio- } \\
\text { do }\end{array}$ & Date & Declinazione del sole & Andamento \\
\hline I & dal $22-I V$ al $21-V I$ & $\mathrm{da}+\frac{23^{\circ} \cdot 5}{2} \mathrm{a}+23^{\circ}, 5$ & solstiziale estivo \\
\hline I i & dal 22 -VI al 23 -VIII & $\mathrm{da}+23^{\circ}, 5 \mathrm{a}+\frac{23^{\circ}, 5}{2}$ & solstiziale estivo \\
\hline II I & dal 24-VIII al 24-X & $\mathrm{da}+\frac{23^{\circ}, 5}{2} \mathrm{a}-\frac{23^{\circ}, 5}{2}$ & equinoziale \\
\hline IV & dal $25 \cdot X$ al $23-X I I$ & . $1 . \quad \frac{23}{2} a-23^{0}, 5$ & solstiziale invernale \\
\hline V & dal 24 XII al 18-II & $\mathrm{da}-23^{\circ}, 5 \mathrm{a}-\frac{23^{\circ}, 5}{2}$ & solstiziale invernale \\
\hline VI & dal I9-II al 2I-IV & $\mathrm{da}-\frac{23^{\circ} \cdot 5}{2} \mathrm{a}+\frac{23^{\circ} .5}{2}$ & equinoziale \\
\hline
\end{tabular}

Il numero dei giorni che presentano l'andamento equinoziale è perciò:

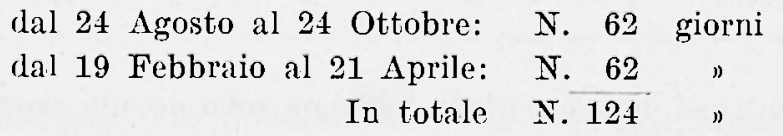

Il numero dei giorni che presentano l'andamento solstiziale estivo è: dal 22 Aprile al 23 Agosto: N. 124 giorni. 
Infine il numero dei giorni che presentano l'andamento solstiziale invernale $\dot{e}:$

dal 25 Ottobre al 18 Febbraio: N. 117 giorni.

L'andamento medio annuo è stato perciò calcolato con lá seguente relazione:

$$
\left.\bar{\Sigma}_{T}=\frac{191}{365}\left(\Sigma_{T}\right)_{\text {eq. }}+\frac{124}{365}\left(\Sigma_{T}\right)_{\text {sol. est. }}+\frac{117}{365} \Sigma_{T}\right)_{\text {sol. suv. }}
$$

I valori cosi ottenuti sono stati riportati nella Tab. IV e l'andamento è disegnato nella Fig. 3. Il minimo viene a trovarsi alle 8.20 (T.U.) ed il massimo alle 22.30 (T.U.).

Tabella IV - ANDaMento Medo anNuo DELla SUPERficie apparente DETLE TERRE EMERSE ILLUMINATE DAL SOLE (in $10^{4} \mathrm{~km}^{2}$ ).

\begin{tabular}{|c|c|c|c|c|c|c|c|}
\hline T.U. & $\Sigma_{T}$ & T.U. & $\Sigma_{T}$ & 'T.U. & $\Sigma_{T}$ & 'T.U. & $\Sigma_{T}$ \\
\hline 0.40 & 2032 & 6.40 & 4988 & 12.40 & 4712 & 18.40 & 2709 \\
\hline 1.20 & 2306 & 7.20 & 5196 & 13.20 & 4550 & 19.20 & 2485 \\
\hline 2.00 & 2606 & 8.00 & 5293 & 14.00 & 4346 & 20.00 & 2234 \\
\hline 2.40 & 2910 & 8.40 & 5284 & 14.40 & 4093 & 20.40 & 1968 \\
\hline 3.20 & 3219 & 9.20 & 5209 & 15.20 & 3809 & 21.20 & 1737 \\
\hline 4.00 & 3555 & 10.00 & 5114 & 16.00 & 3533 & 22.00 & 1593 \\
\hline 4.40 & 3927 & 10.40 & 5024 & 16.40 & 3295 & 22.40 & 1562 \\
\hline 5.20 & 4319 & 11.20 & 4941 & 17.20 & 3097 & 23.20 & $4 \mp 91$ \\
\hline 6.00 & 4688 & 12.00 & 4844 & 18.00 & 2911 & 24.00 & $180_{6}$ \\
\hline
\end{tabular}

$\Sigma$

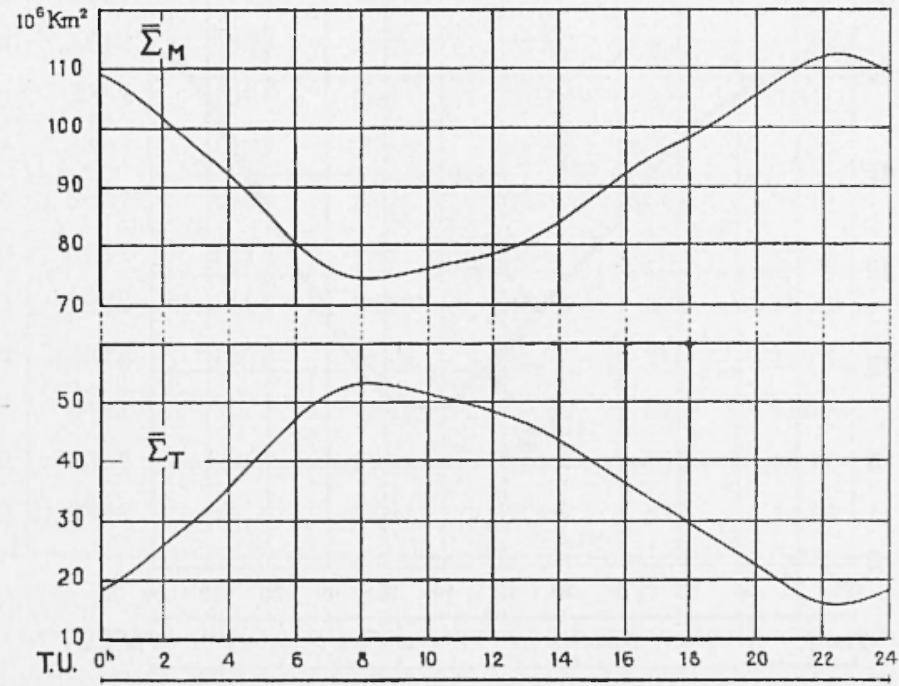

Fig. 3 
Fella Fig. 3 ì riportato anche l'andamento medio annuo delle superfici apparenti dei mari $\bar{\Sigma}_{n}$ illuminati dal sole, facendo al solito la differenza tra l'area della sezione massima della terra e la $\bar{\Sigma}_{T}$.

Abbiamo infine cercato, data la regolarità dell'andamento, una funzione che lo potesse descrivere. Si è visto così che esso può essere ben mppresentato con una funzione cosinussoidale, il cui periodo varia durante il giorno con una legge del seguente tipo:

$$
\begin{array}{rlr}
0 \leqslant t \leqslant 4^{\mathrm{h}} & \omega=15,00-1,25 t & \text { gradi/ora } \\
4^{\mathrm{h}} \leqslant t \leqslant 10^{\mathrm{h}} & \omega=9,52+0,12 t & \text { " " } \\
10^{\mathrm{h}} \leqslant t \leqslant 24^{\mathrm{h}} & \omega)=7,80+0,30 t
\end{array}
$$

" "

La funzione rappresentativa è quindi:

$$
\Sigma_{T}=93,2-18,7 \cos \omega t, \quad\left(10^{6} \mathrm{~km}^{2}\right)
$$

dove $t$ è il tempo solare (espresso in ore) riferito al meridiano di $125^{\circ} \mathrm{W}$ (circa il meridiano di S. Francisco), spostato ciò̀ di $8^{\text {n}} 20^{\prime}$ dal meridiano di Greenwich.

Sella Fig. 4 sono state riportate a tratto pieno la curva relativa a $\Sigma_{r}$ calcolata secondo la[1] e, a trattini, la curva calcolata con la [2].

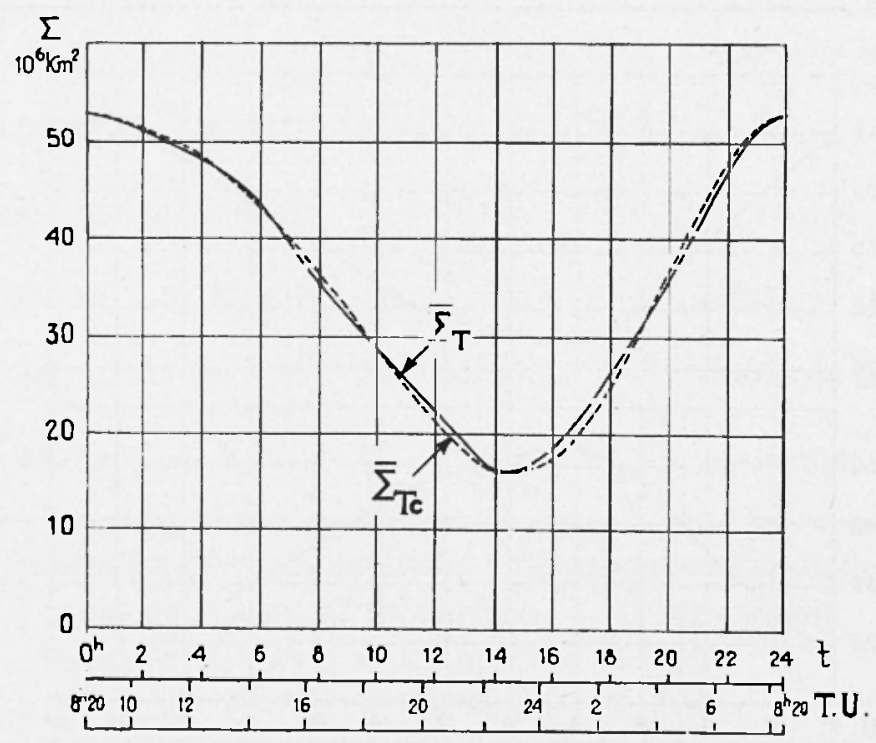

Fig. 4 
Lat superficie apparente delle terre cmerse (a rispettivamente quella dei mari) dà, a meno della costante solare, l'energia che cade sulle regioni coperte dalle terre emerse (e rispettivamente dai mari). Sarebbe però opportuno tener conto del fatto che l'energia che arriva effettivamente al suolo (o alla superficie del mare) viene in parte assorbita dall'atmosfera e questo assorbimento varia al variare dell'altezza zenitale del sole.

Tabella $V$ - SUPERFICIE APPARENTE DELLE TERRE LMLRS ILLUMINATE DAI, SOLE CON ta CORREzIONE PER l'assorbinexto atMosferico (in $10^{\prime} \mathrm{h} \mathrm{m}^{2}$ ).

\begin{tabular}{|c|c|c|c|c|c|c|c|}
\hline 'T.U. & Equinoz. & $\begin{array}{l}\text { Solstizio } \\
\text { estivo }\end{array}$ & $\begin{array}{l}\text { Solstizio } \\
\text { invernale }\end{array}$ & T.l. & Equinoz. & $\begin{array}{l}\text { Solstizio } \\
\text { estivo }\end{array}$ & $\begin{array}{l}\text { Solstizio } \\
\text { invernale }\end{array}$ \\
\hline 0.40 & 1070 & 1631 & 978 & 12.40 & 3138 & 3670 & 2760 \\
\hline 1.20 & 1319 & 1872 & 1163 & 13.20 & 2993 & 3518 & 2635 \\
\hline 2.00 & 1592 & 2147 & 1350 & 14.00 & 2820 & 3344 & 2474 \\
\hline 2.40 & 1856 & 2431 & 1530 & 14.40 & 2636 & 3144 & 2300 \\
\hline 3.20 & 2099 & 2711 & 1690 & 15.20 & 2466 & 2952 & 2133 \\
\hline 4.00 & 2334 & 2984 & 1843 & 16.00 & 2325 & 2789 & 1995 \\
\hline 4.40 & 2575 & 3258 & 2021 & 16.40 & 2219 & 2645 & 1893 \\
\hline 5.20 & 2845 & 3541 & 2222 & 17.20 & 2122 & 2500 & 1812 \\
\hline 6.00 & 3117 & 3830 & $2 \cdot 123$ & 18.00 & 2004 & 2340 & 1713 \\
\hline 6.40 & 3353 & 4097 & 2591 & 18.40 & 1850 & 2168 & 1573 \\
\hline 7.20 & 3534 & 4307 & 2716 & 19.20 & 1641 & 1976 & 1381 \\
\hline 8.00 & 3641 & 4438 & 2795 & 20.00 & 1382 & 1759 & 1152 \\
\hline 8.40 & 3674 & 1478 & 2827 & 20.40 & 1103 & 1545 & 921 \\
\hline 9.20 & 3632 & $4+24$ & 2829 & 21.20 & 867 & 1373 & 734 \\
\hline 10.00 & 3542 & 4292 & 2829 & 22.00 & 722 & 1272 & 630 \\
\hline 10.40 & $34+1$ & 4121 & 2845 & 22.40 & 685 & 1251 & 622 \\
\hline 11.20 & 3352 & 3958 & 2858 & 23.20 & 739 & 1308 & 689 \\
\hline 12.00 & 3258 & 3814 & 2834 & 24.00 & 871 & 1438 & 811 \\
\hline
\end{tabular}

E necessario pertanto apportare a questo calcolo mal correzione, tenendo presente che la radiazione solare viene diminnita di tore $(p))^{s e c} z$, love $p$ è il cocfficiente di trasmissione atmosferical e $z \grave{\mathrm{e}}$ 
l'altezza zenitale del sole. Si è così moltiplicata la superficie apparenet delle terre presenti in ciascuna di quelle regrioni di $10^{\circ} \times 10^{\circ}$, con le quali arovamo precedentemente suddivisa tutta la superficie della terra, per il fattore $(p)^{\text {sec } z}$ : per $p$ si è preso un valore $\left(^{3}\right)$ medio di 0,8 . Sommando tutti questi prodotti si è ottenuta la superficie apparente delle terre illuminate dal sole corretta per l'assorkimento dell'atmosfera. Questa somma è proporzionale, a meno della costante solare, alla quantità di energia che cade in un certo istante sulle terre emerse. In realta le nubi, certamente presenti in qualche regione della terra, possono diminuire notevolmente l'energia totale che arriva sulla terra; ma potremo fare una ipotesi di prima approssimazione supponendo che la nuvolosità sia presente sulle terre emerse e sui mari con una percentuale in media costante. Questa ipotesi porta come conseguenza che la presenza delle nubi non altera l'andamento delle $\Sigma_{T}$ e delle $\Sigma_{M}$.

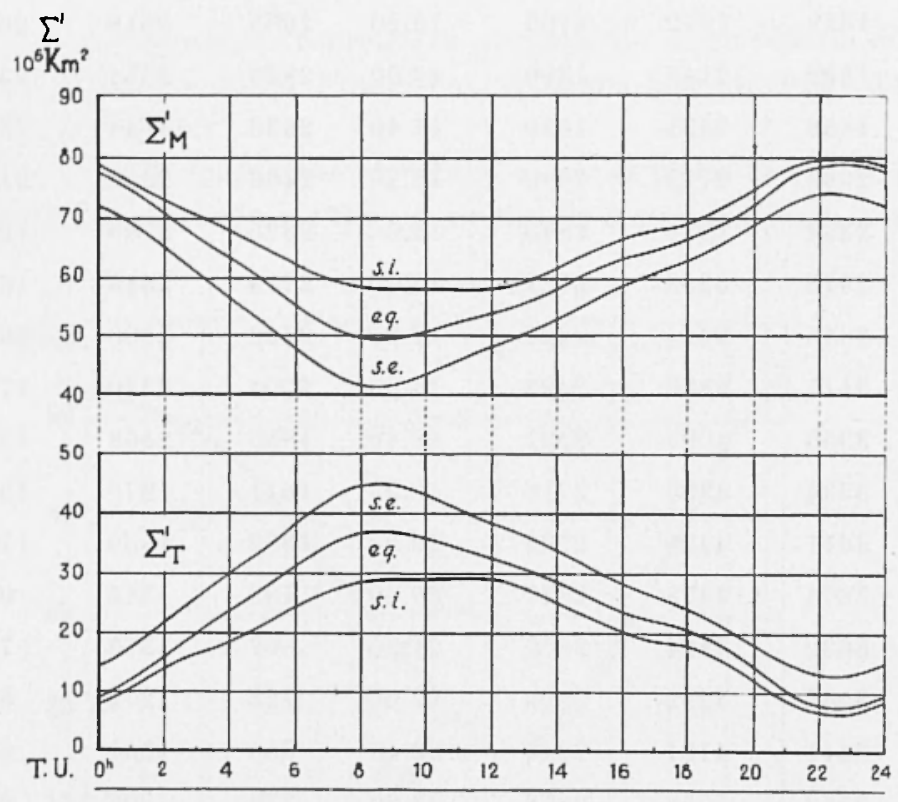

Fig. 5

Nella Tab. V e nella Fig. 5 sono riportati $i$ valori ed il relativo andamento di questa superficie apparente.

I'andamento non differisce sostanzialmente da quello calcolato senza tener conto dell'assorbimento atmosferico, pur presentando una 
variazione percentuale maggiore. I massimi e i minimi per $\Sigma_{T}$ sono stati calcolati analogamente come per la $\Sigma_{r}$ e si sono ottenuti i seguenti valori:

Tabella VI

\begin{tabular}{|c|c|c|}
\hline Periodi & Massino & Minimo \\
\hline Equinozio. . & 8.40 & 22.30 \\
\hline Solstizio estivo & 8.30 & 22.30 \\
\hline Solstizio invernale. . & 11.10 & 22.20 \\
\hline
\end{tabular}

Nella Fig. 5 è stata riportata anche la $\Sigma_{M}^{\prime}$, cioè la superficie apparente dei mari illuminati dal sole corretta per l'assorbimento. Per poterla calcolare si è anzitutto trovata la superficie apparente di tutta la terra corretta per l'assorbimento atmosferico. La superficie totale apparente di ogni sezione della terra di $10^{\circ} \times 10^{\circ}$ è stata moltiplicata per il fattore $(p)^{\text {sec } z}$ e poi si sono sommati tutti questi prodotti. Si è ottenuto così il valore di $8595 \cdot 10^{1} \mathrm{~km}^{2}$ : per differenza con la $\Sigma^{\prime}{ }^{\prime}$ si è trovata la $\Sigma^{\prime}$.

Tabella VII - ANDAMENTo medio anNuo DelLa SUPERfICIE APPARENTE DElle terRe mMerse, ILluminate dal SOle, CON LA CORrezione Per L'assoribimento atmosferico (in $10^{4} \mathrm{~km}^{2}$ ).

\begin{tabular}{|c|c|c|c|c|c|c|c|}
\hline 'T.U. & $\Sigma^{\prime} T$ & 'T.U. & $\Sigma_{T}^{\prime}$ & T. I. & $\Sigma^{\prime} \boldsymbol{T}$ & T.U. & $\Sigma^{\prime} T$ \\
\hline 0.40 & 1231 & 6.40 & 3361 & 12.40 & 3197 & 18,40 & 1869 \\
\hline 1.20 & 1457 & 7.20 & 3535 & 13.20 & 3057 & 19.20 & 1672 \\
\hline 2.00 & 1703 & 8.00 & 3641 & 14.00 & 2887 & 20.00 & 1436 \\
\hline 2.40 & 1947 & 8.40 & 3676 & 14.40 & 2701 & 20.40 & I 195 \\
\hline 3.20 & 2176 & 9.20 & 3644 & 15.20 & 2524 & 21.20 & 996 \\
\hline 4.00 & 2397 & 10.00 & 3568 & 16.00 & 2377 & 22.00 & 879 \\
\hline 4.40 & 2630 & 10.40 & 3481 & 16,40 & 2259 & 22.40 & 857 \\
\hline 5.20 & 2882 & 11.20 & 3400 & 17.20 & $215 \mathrm{l}$ & 23.20 & 916 \\
\hline 6.00 & 3137 & 12.00 & 3311 & 18.00 & 2025 & 24.00 & 1044 \\
\hline
\end{tabular}


Con un procedimento del tutto analogo al caso precedente si ̀̀ calcolato l'andamento medio annuo di $\Sigma^{\prime}$, e per differenza l'andamento medio annuo di $\Sigma^{\prime}{ }_{M}$.

Nella Tab. VII e nella Fig. 6, sono riportati i valori di $\Sigma_{T}^{\prime}$ e gli andamenti di $\bar{\Sigma}_{T}^{\prime}$ e di $\bar{\Sigma}_{M}^{\prime}$.

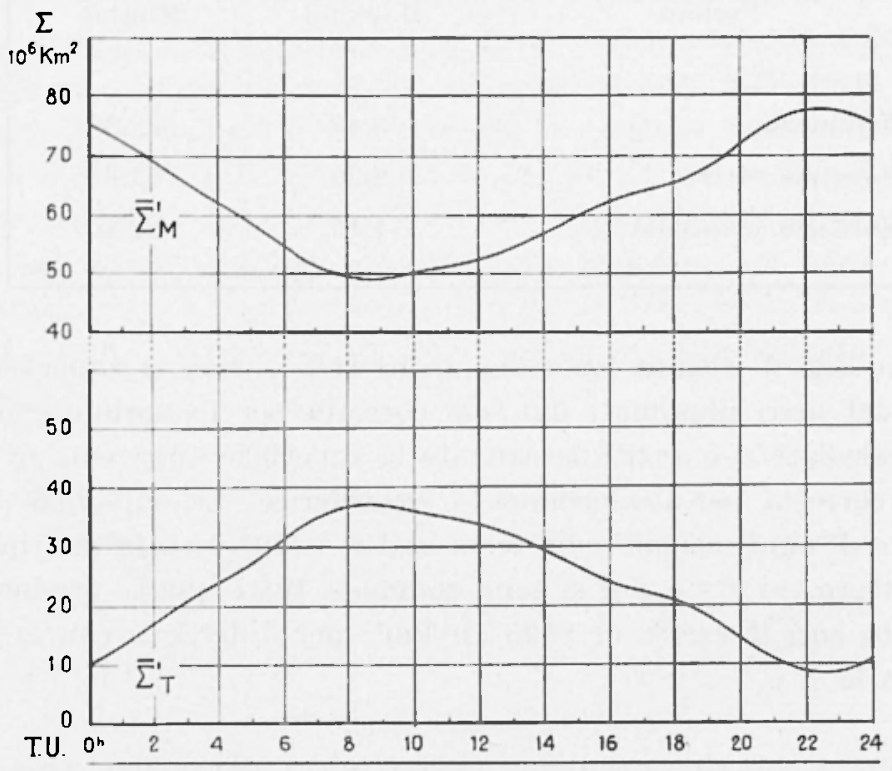

Fig. 6

Abbiamo cercato, come nel caso precedente, una funzione che potesse descrivere l'andamento della $\Sigma_{T}^{\prime}$, e si è trovato che esso può essere rappresentato dalla:

$$
\Sigma^{\prime}{ }_{T}=22, \tau+14,1 \cos \omega t \quad \text { (in } 10^{\circ} \mathrm{km}^{2} \text { ) }
$$

dove $\omega$, variabile durante il giomo è dato dalle seguenti relazioni:

$$
\begin{array}{rlrl}
0^{\mathrm{n}} \leqslant t \leqslant 10^{\mathrm{n}} & \omega=15,00-0,435 \quad t & \text { gradi/ora } \\
10^{\mathrm{n}} \leqslant t \leqslant 16^{\mathrm{n}} & \omega=5,23+0,542 \quad t & " ~ " ~ \\
\mathrm{I} 6^{\mathrm{n}} \leqslant t \leqslant 24^{\mathrm{n}} & \omega=11,70+0,1375 t & " \prime,
\end{array}
$$

e $t$ è il tempo solare (espresso in ore) riferito al meridiano di $130^{\circ} \mathrm{W}$, spostato cioè di $8^{\mathrm{h}} 40^{\prime}$ dal meridiano di Greenwich.

Nella Fig. 7 sono state riportate a tratto pieno la curva relativa a $\Sigma_{T}^{\prime}$ calcolata secondo la [1] - dove al posto delle superfici apparenti 
$\Sigma_{T}$ compaiono le superfici apparenti $\Sigma_{T}^{\prime}$ corrette per l'assorbimento atmosferico - e a trattini la curva calcolata con la [3].

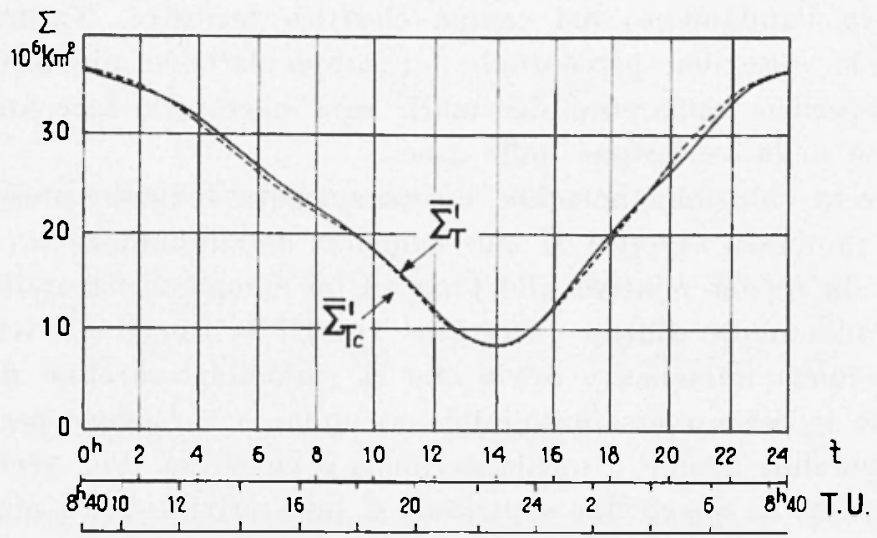

Fig. 7

Prendiamo ora in esame un fenomeno naturale che abbia un andamento universale, per es. il campo elettrico terrestre. Esso presenta $\left({ }^{2}\right)$ un minimo alle $t^{\text {h }}$ T.U. ed un massimo alle $19^{\text {h }} 30^{\prime}$ T.U.: la sua variazione percentuale è del $36 \%$ (v. Fig. 8 ).

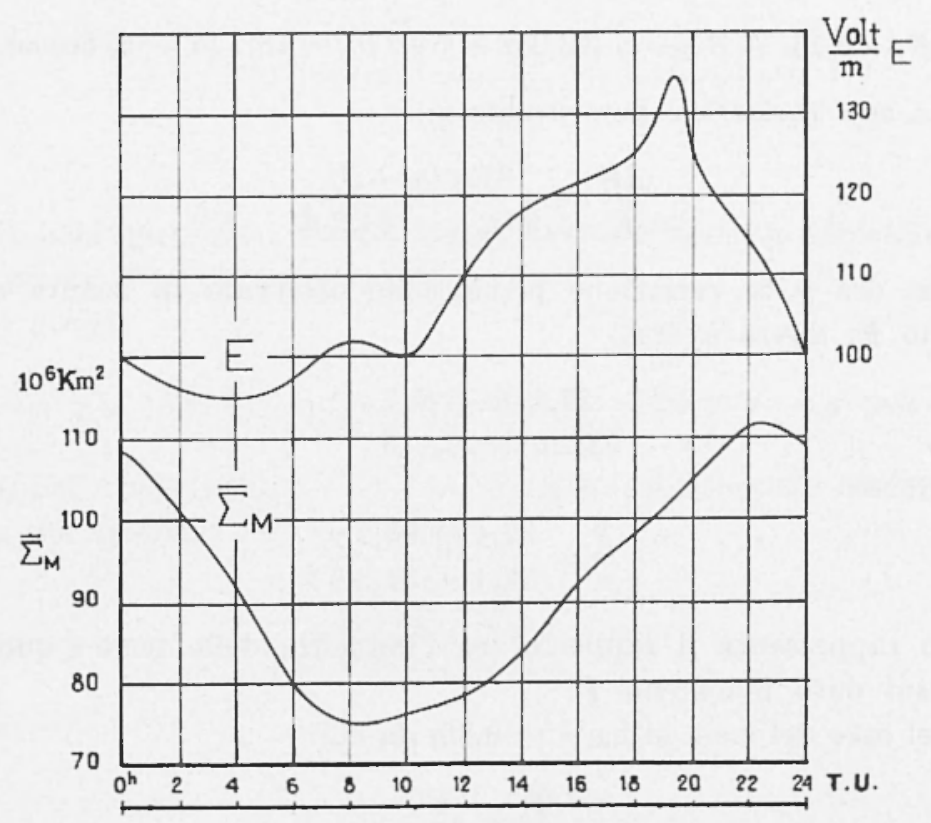

Fig. 8 
Ebbene si vedo subito che facendo slittare di circa 4 ore la curva dell'andamonto della superficio apparente dei mari illuminati dal sole, si ritrova l'andamento del campo elettrico terrestre. Naturalmente, essendo la variazione percentuale del campo elettrico minore di quella della superficie apparente dei mari, sarà necessario fare anche una riduzione della variazione delle aree.

Questa riduzione potrebbe essere spiegata tenendo presente che e stata ammessa l'ipotesi di una influenza dell'illuminazione dei mari liversa da quella relativa alle terre su un fenomeno naturale presentante l'andamento diurno universale. Infatti se i mari e le terre avessero la stessa influenza è ovvio che la variazione sarebbe del tutto nulla; se le terre avessero un'influenza nulla, la variazione pereentuale diurna sarebbe uguale a quella dei mari e viceversa. Per aver un'idea dal rapporto di questi due contributi si può scrivere per i mari e per le terre rispettivamente:

$$
\begin{aligned}
& \Sigma_{M}=93,2-18,7 \cos \omega t \\
& \bar{\Sigma}_{T}=34,2+18,7 \cos \omega t,
\end{aligned}
$$

e indicando con $F$ un qualsiasi fenomeno naturale dipendente da $\Sigma_{T}$ e da $\Sigma_{n}$, sia pure con coefficienti diversi, si ha:

$$
F=\alpha \Sigma_{M}+\beta \Sigma_{T}=(93,2 \alpha+34,2 \beta)-18,7(\alpha-\beta) \cos \omega t .
$$

La sua variazione percentuale è:

$$
\frac{\Delta F^{\prime}}{F^{\prime}}=\frac{3 \tau, 4(\alpha-\beta)}{93,2 \alpha+34,2 \beta}
$$

Sia ora $\gamma$ la variazione percentuale osservata in natura sul fenomeno $F$ : dovrà aversi,

da cui:

$$
\frac{37,4(\alpha-\beta)}{93,2 \alpha+34,2 \beta}=\gamma,
$$

$$
\frac{\beta}{a}-\frac{37,4-93,2 \gamma}{37,4+34,2 \gamma},
$$

questo rappresenta il rapporto tra l'influenza delle terre e quelle dei mari sul dato fenomeno $F$.

Tel caso del c.e.t. si ha $\gamma=0,36$, da cui:

$$
\frac{\beta}{\alpha} \sim \frac{1}{13}
$$


L'ASSORBIMENTO DELLA RADIAZIONE SOLARE SU SCALA PLANETARIA 169

cioè l'influenza dei mari è circa 13 volte quella delle terre emerse.

Nella Fig. 9 è riportata la curva:

$$
f=\bar{\Sigma}_{M}+\frac{1}{13} \bar{\Sigma}_{T}
$$

oltre all'andamento diurno universale del c.e.t.

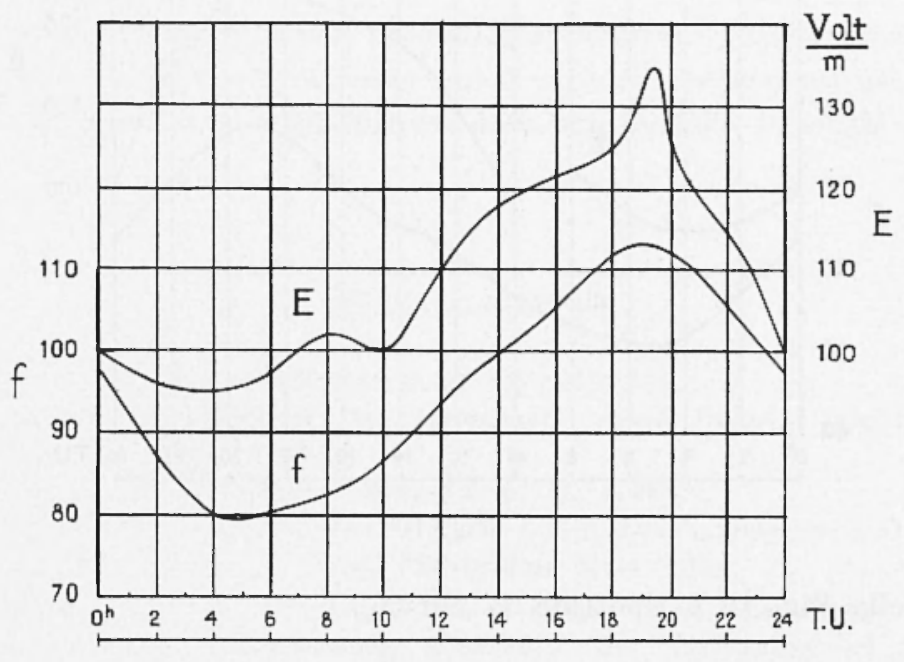

Fig. 9

Un analogo calcolo, tenendo conto dell'assorbimento dell'atmosfera, porta ad un altro valore del rapporto $\beta / \alpha$.

Si ponga:

$$
F=\alpha^{\prime} \Sigma^{\prime}{ }_{1}+\beta^{\prime} \Sigma_{T}^{\prime}=\left(63,3 \alpha^{\prime}+22,7 \beta^{\prime}\right)-28,2\left(\alpha^{\prime}-\beta^{\prime}\right) \cos (\omega t,
$$

da cui, indicando sempre con $\gamma$ la variazione percentuale osservata in natura nel fenomeno $F$, si ottiene:

$$
\frac{28,2\left(\alpha^{\prime}-\beta^{\prime}\right)}{63,3 \alpha^{\prime}+22,7 \beta^{\prime}}=\gamma
$$

da cui:

$$
\frac{\beta^{\prime}}{\alpha^{\prime}}=\frac{28,2-63,3 \gamma}{28,2+22,7 \gamma}
$$


Introducendovi il valor's $\gamma=0,36$ del c.e.t. si ha:

$$
\frac{\beta^{\prime}}{a^{\prime}} \sim \frac{1}{6,7} \text {. }
$$

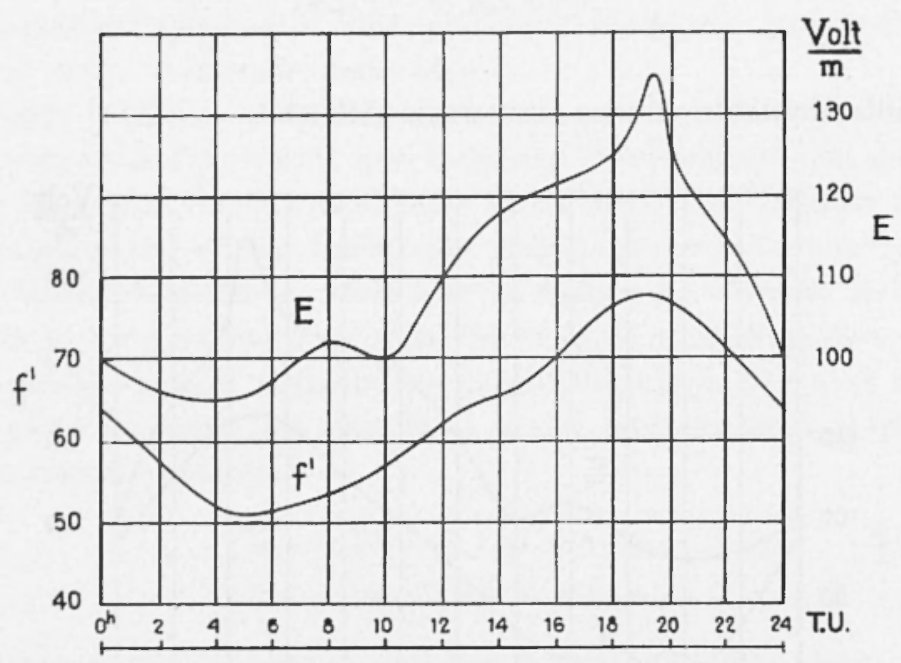

Figr. 10

Solla Figr. 10 è riportata la curva $f^{\prime}$ :

$$
f^{\prime}=\bar{\Sigma}_{M}^{\prime}+\frac{1}{6,7} \bar{\Sigma}_{T}^{\prime}
$$

oltre all'andamento diurno universale del c.e.t.

Se si tiene conto dell'assorbimento dell'atmosfera, si osserva che l'influenza dei mari nel c.e.t. è circa sette volte l'influenza delle terre emerse.

Si può quindi concludere che il c.e.t. sembra essere influenzato istante per istante dall'energia solare assorbita complessivamente dalla terra: infatti le superfici dei mari presentano un albedo molto più piccolo di quello presentato dalle terre emerse. Pertanto il valore del rapporto $\beta^{\prime} / \alpha^{\prime}$ trovato è più attendibile di quello $\beta / a$, in quanto il rapporto dei coefficienti di assorbimento (") non raggiunge certamente il valore di 13, ma è crtament. inferiore a 6 . Infatti il valore di questo rapporto, è difficile a prefissarsi in quanto l'albedo delle terre emerse è molto vario, dipendendo notevolmente dalla natura del suolo. Esso può andare dal $3 \%$ per i prati fino a circa 1 ' $85 \%$ per le superfici rico. perte da neve $\left({ }^{3}\right)$. Bisogna inoltre tener conto che, oltre all'albedo, 
esiste un altro elemento di primaria importanza nel bilancio termico, e cioè lo scambio di energia tra terra o mare e l'atmosfera.

Pertanto si può concludere che, pur non conoscendo nei particolari il meccanismo con il quale l'energia del sole, che cade complessivamente sulla term, possa influenzare il c.e.t., Vandamento dell'energia totale assorbita complessivamente dalla terra e quello del c.e.t. sembrano del tutto analoghi, demunciando un qualche effet to dell'una sull'altra.

Inoltre ci è parso molto interessante mostrare questa correlazione in quanto, a nostro parere, essa potra portire certamente un contributo allo studio del meccanismo della circolazione generale dell'atmosfiera $\left({ }^{5}\right)$.

\section{BIBIIOGRAFIA}

(') Alzirecil F., Amm. Meleorol., 2, p. 129, (1949).

Loxpox J., A Study of the almospheric lleal Balance. Dept. Mot., New York Univ., (1957).

HovillTos II. G., Journ. Mel., 11. p. II, (1954).

BüYко M. J. - Koxdratev K. Y., in Research in (ieophysies, di Odishaw H., 2. p. 529, M.I.T. Press, Cambridge, Mass. 1964.

(2) Whippl, F. J. W., Scrase F. J., Geophys. Mem. Lond., 68. (1936). Byers H. R., Thunderstorm Electricily. The University of Chieago Press. (1953).

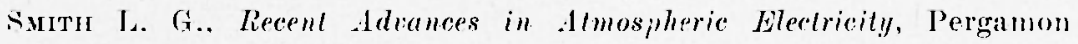
Press, London, (1958).

(3) Roulleat J., Trociox R., Météorologie gémérale. Tome I, Ganthier-Villars. Parik, (1952).

(1) Axgströм A., (ieogr. Ann., 7, p. 323, (1925).

Goove J. P., Goode's School Allas. Rand IL Nally Co. Now York, (1947).

Fritz s., Journ. Wel., 6. p. 277, (1949).

Axgströ̀ A., Tellus, 14, p. 435, (1962).

(5) Starr V. P., Compendium of Weleorology, Am. Met. Soc., p. 1017, Boston. White R. M.. Trans. Am. Geophys. Union, 32, p. 391, (1951).

LetTau II., Arehiv jür Met. (ieophys. und Bioch.. 7. p. 133. (1954).

Rao Y. P', Quarl. Journ. of Roy. Mel. Son., 86. p. 156, (1960). 